\title{
Distilling Nanoscale Heterogeneity of Amorphous Silicon using Tip-enhanced Raman Spectroscopy (TERS) via Multiresolution Manifold Learning
}

Guang Yang ( $\nabla$ yangg@ornl.gov )

Oak Ridge National Laboratory

Xin Li

Oak Ridge National Laboratory

Yongqiang Cheng

Oak Ridge National Lab

Mingchao Wang

Monash University

Dong Ma

Oak Ridge National Laboratory https://orcid.org/0000-0003-3154-2454

Alexei Sokolov

Oak Ridge National Laboratory

\section{Sergei Kalinin}

The Center for Nanophase Materials Sciences, Oak Ridge National Laboratory, Oak Ridge, TN 37831

https://orcid.org/0000-0001-5354-6152

\section{Gabriel Veith}

Oak Ridge National Laboratory https://orcid.org/0000-0002-5186-4461

Jagjit Nanda

Oak Ridge National Laboratory

\section{Article}

Keywords: TERS, amorphous silicon, structural heterogeneity

Posted Date: July 14th, 2020

DOI: https://doi.org/10.21203/rs.3.rs-38466/v1

License: (1) (1) This work is licensed under a Creative Commons Attribution 4.0 International License.

Read Full License 
Version of Record: A version of this preprint was published at Nature Communications on January 25th, 2021. See the published version at https://doi.org/10.1038/s41467-020-20691-2. 


\section{Abstract}

Accurately identifying the local structural heterogeneity of complex, disordered amorphous materials such as amorphous silicon (a-Si) is crucial for accelerating technology development. However, shortrange atomic ordering quantification and nanoscale spatial resolution over a large area on a-Si have remained major challenges and practically unexplored. We resolve phonon vibrational modes of a-Si at a lateral resolution of $20 \mathrm{~nm}$ by tip-enhanced Raman spectroscopy (TERS). To project the high dimensional TERS imaging to a low dimensional (i.e. 2D) manifold space and categorize a-Si structure, we developed a multiresolution manifold learning (MML) algorithm. It allows for quantifying average Si-Si distortion angle and the strain free energy at nanoscale without a human-specified threshold. The MML multiresolution feature allows for distilling local defects of ultra-low abundance $(<0.3 \%)$, presenting a new Raman mode at finer resolution grids. This work promises a general paradigm of resolving nanoscale structural heterogeneity and updating domain knowledge for highly disordered materials.

\section{Introduction}

Silicon is central for a gamut of applications including large-scale integrated electronic circuits, ${ }^{1}$ photonics, ${ }^{2}$ photovoltaics ${ }^{3,4}$ and energy storage units. ${ }^{5,6,7}$ It is well known that the essences of Sibased materials, including optical, electrical ${ }^{8}$ properties and nuclear spin, ${ }^{9}$ are highly related to their atomic structures. Ever since Russell reported the first observation of the first-order inelastic Raman scattering in a Si single crystal, ${ }^{10}$ Raman spectroscopy has been intensively used to investigate the Si crystal structure, ${ }^{11}$ phonon dispersion, ${ }^{12}$ electronic states, ${ }^{13}$ local stress and strain, ${ }^{14,15}$ and thermal properties, ${ }^{16}$ which are integral to the performances of silicon-based devices. Despite its versatility, the applications of micro-Raman spectroscopy to characterize the submicron-to-nanoscale chemistry and physics are severely restrained by the intrinsic diffraction limit of the visible light (i.e. $>200 \mathrm{~nm}$ ) based on Abbe's law. ${ }^{17}$ Tip-enhanced Raman spectroscopy (TERS) provides an apertureless means of mapping Raman scattering at nanometer scale $(\sim 10 \mathrm{~nm})$ in-sample plane. ${ }^{18,19}$ This is based on that the surface plasmon (i.e. enhanced electromagnetic field or EM-field) resides at the metallized tip apex of a scanning probe microscope (SPM), providing a localized "hot-spot" underneath the SPM tip. Consequently, the Raman scattering of the sample within the hot-spot is largely increased, unraveling surface vibrational modes with a nanoscale lateral resolution. ${ }^{20}$ Sun et al. first reported the successful TERS study on a Si wafer. ${ }^{21}$ With a $50 \%$ increase in the TERS intensity with respect to Raman, they were able to construct the TERS mapping of the Si transverse optical (TO) mode $\left(520 \mathrm{~cm}^{-1}\right)$ at $\sim 100 \mathrm{~nm}$ resolution. Later, upon optimization of the polarization conditions, Sokolov et al. improved the ratio of the near-field Raman intensity and far-field Raman intensity by more than one order of magnitude, ${ }^{22}$ hence being able to carry out the nano-Raman analysis of the crystal $\mathrm{Si}$ at a $\sim 20 \mathrm{~nm}$ lateral resolution.

Despite great efforts in deciphering the nanoscale vibrational structures on the Si surface using TERS, the research focus has only been on crystal $\mathrm{Si}$ (c-Si) so far. Due to the highly symmetric diamond cubic crystal structure of c-Si, its inelastic Raman shift carries the information reflecting optic phonon energy 
only at the center (i.e. Г-point) of the first-order Brillouin zone (BZ). ${ }^{11,23}$ Therefore, the spectral variation on two adjacent sampling points is rather vague on TERS mapping.

Different from its c-Si counterpart, the energy states of amorphous silicon (a-Si) vary in the first order BZ, hence resulting in several convoluted Raman active modes. Due to k-selection rule breaking down for amorphous materials, the TERS spectrum taken at each sampling point roughly corresponds to the phonon density of states (PDOS), thereby capable of carrying local information of the a-Si. ${ }^{11}$ However, lacking the long-range atomic ordering and symmetries, to quantify the structure of amorphous materials, such as a-Si remains a long-stand challenge. This is especially true for a large TERS dataset gathered from a-Si over a large scanning area, which contains spectra collected from adjacent sampling points of nanometers away sampling points. Accordingly, it is almost impossible to implement a manual exploration over the structural metrics of the a-Si, let alone mining essential information of a miniature structure embedded in such a large dataset. In addition, complex interactions between the tip-induced EM-field and Raman scattering tends to restrict the use of traditional linear dimension reduction techniques, such as principal component analysis (PCA) (e.g. it becomes difficult to extract physical information from unmixed components). ${ }^{18}$ This explains why the nanoscale structural heterogeneity of a-Si or other amorphous materials has been much underexplored by TERS so far.

We herein illustrate a multiresolution analytical framework based on graph-analytics and an unsupervised manifold learning algorithm to facilitate identifying the nanoscale structure of a-Si thin film. High-dimensional hyperspectral TERS mapping on the a-Si thin film comprises thousands of TERS spectra. The multiresolution manifold learning (MML) algorithm projects the TERS mapping to a low dimensional (i.e. 2D) manifold space, thereby allowing for ease of straightforward data visualizations and structural categorization. Unlike traditional manifold learning methods targeting solely on overlaying the prior-known labels over the manifold points, ${ }^{24}$ the $M M L$ proposed here does not require any prior bias regarding the material structure and instrumental modality. ${ }^{25,26}$ Benefiting from this nature, the underlying a-Si structural and physical properties, such as the average Si-Si distortion angle and the strain free energy can be quantified without a human-specified threshold at a lateral spatial resolution of $20 \mathrm{~nm}$. Further, the multiresolution feature of the MML algorithm allows for extracting child clusters at the finer resolution grid, which carries structural characteristics of minor abundance $(<0.3 \%$ of the sampling points) that would have been hidden in the large dataset. It thus enables us to discover a new Raman mode ascribed to highly disordered $\mathrm{O}_{\mathrm{x}}-\mathrm{Si}-\mathrm{H}_{\mathrm{y}}$ vibrations on a-Si surface that has never been reported before. The identification of such a new vibrational Raman mode was further validated through inelastic neutron scattering and density functional theory (DFT). Although a-Si was used as a model, the integrated workflow proposed is readily available for nanoscale structure-property correlation for other highly disordered materials in general.

\section{Results And Discussion}


The strategy of effectively employing TERS hyperspectral imaging and the MML to elucidate the local structure of the a-Si is schematically illustrated in Fig. 1. Briefly, TERS combines the scanning probe microscopy (SPM) and the Raman spectroscopy technique, enabling the spectral acquisition at a nanoscale lateral resolution. This is due to that the surface plasma resonance (SPR) on a noble metal (such as Ag) coated tip apex upon laser illumination leads to a greatly enhanced EM-field or a hot spot in the gap between the tip and sample surface ( $<5 \mathrm{~nm}$ in depth) (Figure S1(b)). ${ }^{18}$ The Raman scattering cross-section from the analyte in the hot spot is boosted, contributing to the far field enhanced Raman scattering (i.e. TERS spectrum). The area of a typical hot spot on the tip apex is on the scale of $10 \mathrm{~nm}$, thereby breaking the light diffraction limit of the standard confocal micro-Raman spectroscopy. ${ }^{18,22}$ However, the routine TERS mapping composes of innumerable spectra, precluding an easy insight of the underlying structure. This is particularly true for amorphous materials. Therefore, it necessitates a more statistical data processing method capable of implementing batch process of the large quantity TERS spectra to efficiently obtain the structural features within the scanned area.

To explore relationships among all the high dimensional TERS spectra detailing the a-Si local structures, we first construct the nearest neighbor (NN) graph by calculating pairwise distances. For straightforward exploration and visualization purpose, the low dimensional (2D/3D) manifold layout for the NN graph was estimated by solving a principal probability model (details of graph construction and manifold layout can be found at Method section). Clustering can be subsequently performed on the lowdimensional manifold to underpin the intrinsic structure within the manifold that corresponds to the material structure heterogeneity. To better partition intrinsic manifold clusters (facilitating clustering tasks), Li et al. recently proposed a Graph-Bootstrapping procedure 25,26 that iteratively reconstructed the NN graph based on previous manifold positions and then recalculated manifold coordinates based on the reconstructed NN graph. The projected low dimensional manifold clusters represent featured spectral property of the materials, thereby allowing for gaining insights of latent material structures via external validations such as first principle theories. This, in turn, benefits a future experimental design with "human-reasoning" for gaining deeper structure-property relationship of the materials (Fig. 1).

The characteristic TERS spectra obtained from the a-Si surface is shown in Fig. 2(a). All spectra contain a sharp peak located at $520 \mathrm{~cm}^{-1}$, ascribed to the first-order TO mode for the crystal silicon derived from the TERS (Figure S3). For c-Si, only the zone-center TO mode is detectable based on the excitation of the visible Raman laser to the lattices of the diamond structure, with the Si-Si bond angle of $109.5^{\circ} .{ }^{10,11,27}$ Notably, the coexistence of the a-Si and c-Si TO modes in Fig. 2(a) does not indicate that the a-Si is partially crystalized on the surface. ${ }^{28} \mathrm{~A}$ further inspection on the a-Si surface using confocal microRaman spectroscopy with a much higher laser power did not reflect the existence of the c-Si TO band at $520 \mathrm{~cm}^{-1}$ (Figure S3). Instead, a few broad Raman bands were featured in several frequency regions, indicating that the sputtered-silicon in this study is in totally amorphous state. The broadening of the several Raman bands results from the loss of the long-range translational symmetry and corresponding reciprocal lattice of the $\mathrm{c}-\mathrm{Si}$, which allows for detection of the entire phonon density of the states (DOS) across the whole first BZ zone reflected by the Raman spectra. ${ }^{29}$ Though it is inappropriate to correlate 
the traveling wave vectors to the phonon feature in amorphous solids, we assign the a-Si TERS bands to

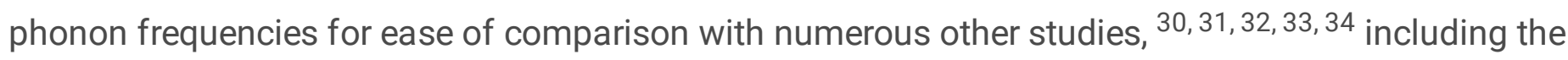
modes of the longitudinal-acoustic $\left(\mathrm{LA}, 312 \mathrm{~cm}^{-1}\right)$, longitudinal-optical $\left(\mathrm{LO}, 400 \mathrm{~cm}^{-1}\right)$, transverse-optical (TO, $473 \mathrm{~cm}^{-1}$ ) and transvers-acoustic (TA, not shown here), respectively. ${ }^{30}$ The second-order phonon modes (denoted as 2LA and 2TO) of either a-Si or c-Si tip are also observable (Fig. 2(a)). The intensity of the second order phonon modes is generally low, perhaps due to the off resonance of the laser frequency with the a-Si direct band gap. ${ }^{35}$ Due to the low signal-to-noise ratio, such second order phonon modes are extremely difficult to be distinguished in standard Raman spectroscopy (Figure S3(b)) on a-Si, corroborating the essential role of TERS in this study.

The TERS mapping is able to provide an overview of the intensity distribution of a given vibrational mode. The normalized intensity (at $520 \mathrm{~cm}^{-1}$ for TO mode) of each vibrational peak is quantified by the color bar with the correspondence mapping representing the abundance of each chemical moiety. Figure 2(b) presents the relative intensity distribution of the a-Si TO mode (approximately centered at $473 \mathrm{~cm}^{-1}$ ). The TERS spectra taken from two spots (denoted as A and B) $\sim 80 \mathrm{~nm}$ apart from each other exhibit different TO mode intensity (Fig. 2(a)). This clearly indicates that the a-Si surface phonon mode unveiled by TERS expresses nanoscale heterogeneity. TERS mapping of the a-Si 2LA mode is shown in Fig. 2(c). Clearly, the feature of the intensity distribution of 2LA mode differs from that of the TO mode shown in Fig. 2(b), demonstrating the highly heterogeneous feature of the phonon modes on a-Si surface. It is also manifested the spectrum taken from Point $C$ on the TERS map shows a larger 2LA mode intensity than those from Points A and B, but the TO band intensity is lower than the latter two (Fig. 2(a)). The a-Si 2TO mode at $943 \mathrm{~cm}^{-1}$ has a favorable distribution on the upper side of the scanned region (Fig. 2(d)), different from the distribution of the above-mentioned phonon modes. The TERS mapping generated based on a single mode intensity clearly demonstrate that i) TERS is capable of depicting the a-Si phonon mode in nanoscale, and ii) the abundance of different a-Si phonon modes is highly heterogeneous and varies across the scanned area.

The mapping analysis based on the single variant method (i.e. intensity of a single TERS band) insufficient to lead to a comprehensive understanding of the surface structure of the a-Si. ${ }^{18}$ Analysis based on an individual TERS band by its nature fails to capture the overall spectral pattern of TERS mapping. To retain the global features embedded in the full set of TERS spectra, we then performed manifold learning and clustering of TERS spectral dataset (see details in method section). Figure 3(a) is the bootstrapped manifold layout of a set of 2500 TERS spectra, with evolution of manifold layouts during Graph-Bootstrapping iteration procedure shown in Figure S4. The 2500 TERS spectra can be categorized into seven clusters (Fig. 3(a)) in the 2D manifold space. Here we denote these clusters as "parent clusters" to differentiate from the "child clusters" derived at finer resolution grids in manifold space (see Fig. 5). The mean TERS spectrum of each cluster is shown in Fig. 3(b). An immediate observation on Fig. 3(b) is that the TO band at $\sim 470 \mathrm{~cm}^{-1}$ intensity varies among clusters. The intensity of the second order phonon peaks also varies, although not much obvious as the TO mode. To quantify the analysis on the TERS bands below $600 \mathrm{~cm}^{-1}$, we implemented peak deconvolution of the overlapped 
phonon vibrational modes of a-Si, including LA, LO, TO modes and c-Si TO mode. A TERS peak deconvolution example is given in Fig. 3(c) based on the spectrum of cluster 0 . The atomistic level local structure of a-Si random network is characterized by the Si-Si bond angle and length distortion $(\Delta \theta),{ }^{33} \mathrm{Si}$ ring topology (e.g. 5 or 6 membered rings), and voids. ${ }^{36}$ The inelastic Raman scattering reflects the full vibrational density-of-states (DOS). ${ }^{37,38}$ Therefore, the local structural change of the a-Si network can be probed by the vibrational frequency change in Raman spectroscopy. Also given that the spring constant for Si-Si bond stretching is much larger than that for bond bending, 39,40 bond length distortions are shown to contributes only $1 \%$ to the structural gap between a-Si and c-Si comparing with that of the bond angle distortion. ${ }^{41,42}$ For TERS measurement, it is only sensitive to $<5 \mathrm{~nm}$ a-Si on surface (Figure S1). Another major contribution towards the structural variation of a-Si also comes from the locally under- or over- coordination of the a-Si network. ${ }^{43,44}$ However, as noted by an early study, the dangling or floating Si bonds resulted from the under- or over- coordination counts from roughly $1 \%$ of the free energy associated to the angle distortion. ${ }^{41}$ In this context, we focus on the correlation between the average bond angle distortions $(\Delta \theta)$ and the vibrational frequencies $(\omega)$ of the TERS spectra on a-Si. Here the average bond angle distortion is defined the Si-Si angle difference than $109.5^{\circ}$, representing the bond angle in a tetrahedral repeating unit in c-Si.

Using a semiexperimental approach, Vink et al. ${ }^{34}$ correlated the TO mode vibrational frequency (in $\mathrm{cm}^{-1}$ ) to the average bond angle distortions, $\Delta \theta$ (in angular degree), linearly as

\section{$\omega_{T O}=-2.5 \Delta \theta+505.5$}

Vink's computational model was built based on a so-called activation-relaxation technique (ART), 45,46 which yields close-to experimental a-Si atomic configurations. Eq. 1 was found to agree reasonably well with experimental values. Although it is generally accepted that the linear correlation between the feature (i.e. the peak center and width) of the TO Raman peak with $\Delta \theta, 33,34,38,47,48$ there still lacks a designative quantitative agreement among all correlations between the two. Here we do not intend to seek a more accurate correlation between the center of the TO mode and the $\Delta \theta$, but rather to show that the TO modes measured by TERS can be correlated to the a-Si local structure represented by $\Delta \theta$. We first rearranged the Raman shift center of the TO mode taken from different clusters in a declining order as shown in Fig. 3(d). Using Eq. 1, we can extract the local distortion angle (marked on Fig. 3(d)), ranging between 13 ${ }^{\circ}$ and $15^{\circ}$. As noted by Beeman et al., ${ }^{48}$ the absolute minimum distortion angle, $\Delta \theta$ was $6.6^{\circ}$ for a continuous a-Si network. Here, the local a-Si angular distortion angles taken from different regions are greater than $13^{\circ}, 12 \%$ of the $109.5^{\circ}$. The maximum distortion angle value was found to be $15^{\circ}$ for Cluster 6 (Fig. 3(d)). This corresponds to the a-Si TO mode centered at $468 \mathrm{~cm}^{-1}$, close to the lower limit of the DOS of the optical branch for a-Si. ${ }^{32}$ Therefore, the model a-Si thin film used here features a highly disordered random a-Si network on the surface. ${ }^{33}$ The calculated strain energy based on the distortion 
angle is close to the upper limit of that reported by Tsu et al. ${ }^{33}$, representing a highly strained a-Si surface (Supporting Information).

So far, we have shown that the surface structure of a-Si explored by TERS can be identified by the MML algorithm without a human-defined threshold, which is meaningful to quantify the "poorly-defined" a-Si random network. To solidify this finding, we compare the experimental TERS spectra with the mean TERS spectrum derived from each parent cluster in Fig. 4(a). For each cluster, the mean TERS spectrum closely matches with its nearest neighbor, with mean absolute error percentage $<5 \%$. In addition, the pairwise Euclidean distance between the mean TERS spectrum and every as-measured TERS spectrum at each pixel is used to check the variance in each cluster more quantitatively. More specifically, the spatial distribution of variance in the material space can be visualized by the similarity loadings (inversion of pairwise Euclidean distances) in Fig. 4(b). For the similarity loading of each cluster, a pixel with higher intensity represents a higher similarity between the corresponding experimental TERS spectrum and the mean cluster TERS spectrum.

We note that the black singular patches in the similarity loadings (marked by yellow circles in Fig. 4(b)) indicate the local TERS spectra different from their surrounding area. To identify these singularities, we performed sub-clustering within each parent cluster by MML. As shown in Fig. 5(a), Parent Cluster 2 was divided into 9 child clusters at a finer resolution grid. An immediate observation is that Child Cluster 8 has a distinguished TERS peak centered at $2435 \mathrm{~cm}^{-1}$ (Fig. 5(b)). A closer exploration on the abundance distribution of the TERS spectra (Fig. 5(c)) indicates that only 7 out of 2500 spectra represented by Child Cluster 9 have the feature of the $2435 \mathrm{~cm}^{-1}$ band. This manifests that the characterization strategy developed in this study is sensitive to $<0.3 \%$ abundance of a structural minority embedded in a large data set. A further inspection on all other singular points shown in the similarity loading maps in Fig. 4 does not reflect TERS spectra carrying any physical meaning. For example, Patent Cluster 0 was subcategorized into 6 child clusters at the finer resolution grid (Fig. 5(d-f)), with the Child Cluster 1 exhibiting a sharp spike at $3245 \mathrm{~cm}^{-1}$. This spike stems from the cosmic ray commonly seen in Raman spectroscopy. Therefore, it necessitates a careful inspection on all stemmed spectra analyzed by the multiresolution method, emphasizing that the domain knowledge human resonating is an essential link in Fig. 1.

The assignment of the $2435 \mathrm{~cm}^{-1}$ TERS band is not intuitive (denoted as X-mode for now), since no fundamental Si-Si vibrational modes exist in the vicinity of this frequency. It is thus reasonable to assume other surface functional groups present on a-Si surface. Noting that the a-Si thin film was RF sputtered in an inert (i.e. argon) atmosphere in the current study, the as-sputtered a-Si surface should be enriched in the unbounded Si atoms. Once exposed to air, we expect the components of the air instantaneously react with dangling Si bonds. Further neutron scattering experiment indicates the presence of the protonated spices on a-Si. As shown in Fig. 6(a), the first confirmation of the proton presence in a-Si sample is the plot of the structure factor, S(Q) (Q: scattering vector), with the contributions from both the coherent scattering and incoherent scattering of the sample itself. ${ }^{49}$ The anisotropic incoherent scattering cross 
section of the proton is $80.26,>37$ times of the Si coherent scattering cross section at 2.163 (inset in Fig. 6(a)). ${ }^{50}$ Therefore, $S(Q)$ plot exhibits a broad background shown in Fig. 6(a) due to incoherent scattering from $\mathrm{H}$. The reactions between the air water and dangling Si bond results in silane- and siloxane- type moieties on a-Si (see Supporting Information).

The simplest protonated Si compound is $\mathrm{Si}-\mathrm{H}$. The reported Raman scattering center for monohydrate (SiH) ranges between $2030 \mathrm{~cm}^{-151}$ and $2090 \mathrm{~cm}^{-1} .{ }^{52}$ Binding more protons to Si leads to blueshift of the $\mathrm{Si}-\mathrm{H}$ stretching mode, with the maximum frequency found for hydrogenated sputtered $\mathrm{Si}-\mathrm{H}_{4}$ compound at $2189 \mathrm{~cm}^{-1} .{ }^{52}$ Given that the surface selection rule complied by TERS differs from those for IR and Raman, ${ }^{18}$ it is difficult to precisely predict the active TERS vibrational modes for $\mathrm{SiH}_{\mathrm{x}}$ compounds in this frequency region.

Inelastic neutron scattering spectroscopy (INS) allows for measuring the vibrational modes in the absence of the selection rules for hydrogen atom, ${ }^{53}$ thereby exhibiting a comprehensive picture of all possible vibrational modes on a-Si sample used here. Figure $6(\mathrm{~b})$ shows an INS spectrum of the a-Si sample. To unambiguously assign the vibrational modes, we further performed density functional theory (DFT) calculation on a surface hydrogenated a-Si, assuming a "water splitting" mechanism (see supporting information) to form the $\mathrm{Si}-\mathrm{H}$ and $\mathrm{Si}-\mathrm{OH}$ functional groups on a-Si surface (Fig. 6(b) inset). The existence of the $-\mathrm{H}$ and $-\mathrm{OH}$ functional groups in the a-Si sample is validated by the excellent agreement between the experimental INS spectrum and that calculated by DFT in Fig. 6(b). The most distinguished proof of the Si-H bond presence is the $\mathrm{Si}-\mathrm{H}$ bending mode centered at $615 \mathrm{~cm}^{-1}$. The presence of the $\mathrm{Si}-\mathrm{OH}$ groups is evidenced by the $\mathrm{Si}-\mathrm{OH}$ twisting bands centered at $869 \mathrm{~cm}^{-1}$ from INS spectrum. ${ }^{54}$ Intriguingly, both experimental and DFT calculated INS spectra show a broad band at around $2430 \mathrm{~cm}^{-1}$, assigned to the 4-phonon overtone of the Si-H bending mode. In fact, the Si-H stretching vibrational mode (frequency as $v$ ) is closely related to the electronegativity of the near-neighbor surroundings, with a simplified induction model formulated as ${ }^{55}$

\section{$v=v_{o}+b \sum X_{A}$,}

where $\mathrm{v}_{\mathrm{O}}$ and $\mathrm{b}$ are empirically derived constants and $\mathrm{X}_{\mathrm{A}}$ is defined as the stability-ratio (SRX) electronegativity. ${ }^{56}$ The values of $\mathrm{v}_{\mathrm{o}}$ and $\mathrm{b}$ were found to be 1741 and 34.7 for molecular compounds, respectively. ${ }^{55}$ The sum is over three neighbors, assuming tetracoodination among the neighboring atoms with the central $\mathrm{Si}$. Different atomic species have various values of $\mathrm{SRX}$, namely $\mathrm{X}_{\mathrm{Si}}=2.62, \mathrm{X}_{\mathrm{H}}=$ 3.55 , and $\mathrm{X}_{0}=5.21 .{ }^{54}$

In this context, the Si-H stretching mode can further blueshift to above $2250 \mathrm{~cm}^{-1} .{ }^{54,57}$ Based on Eq. 2, the highest possible frequency for $\mathrm{Si}-\mathrm{H}$ stretching mode calculated is $2283 \mathrm{~cm}^{-1}$ for $\mathrm{O}_{3}-\mathrm{Si}-\mathrm{H}$ type compound. However, this value is still $152 \mathrm{~cm}^{-1}$ lower than the X-mode shown in TERS spectrum in 
Parent Cluster 2, Child Cluster 8 (Fig. 5(b)). Given that the a-Si thin film is highly disordered on the surface intrinsically, we reason that there possibly exists overly coordinated surface Si atoms of low abundance. We herein define $X_{e}$ as the excess SRX electronegativity contributed from the excess coordination $(>4)$ to the $\mathrm{Si}$. For the case of $\mathrm{X}$-mode of the TERS band, $\mathrm{X}_{\mathrm{e}}$ was calculated at 4.4, close to the reported value for the suboxide silicon as an effective media for a-Si. ${ }^{54}$ In this case, the central Si may form the orthosilicate-type compound with the oxygen in surrounding suboxide silicon. Thus, the large blueshift of the X-mode TERS band than generally reported values stems from overcoordination of the Si-H with the surrounding suboxide $\left(\mathrm{O}_{x}-\mathrm{Si}-\mathrm{H}_{\mathrm{y}}, \mathrm{x}+\mathrm{y}>4\right)$. Since in the same frequency region, as a bulk sensitive spectroscopy technology, INS shows only a broad shoulder (Fig. 6(b)), the abundance of the overcoordination Si compound should thus be critically low. It is therefore worth emphasizing that the existence of the trace amount $(<0.3 \%)$ of the surface defects can only be validated by the ultra-surface sensitive technology, TERS, with the structural information distilled by the MML algorithm.

\section{Conclusions}

We successfully demonstrate that the nanoscale structural heterogeneity of amorphous Si can be identified and quantified by the synergy between TERS hyperspectral imaging and an unsupervised machine learning-based manifold method. Straightforward clustering and visualization of the manifold structure enable the detection of highly localized conformational changes of a-Si at atomistic level, reflecting the underlying structural and physical essences of the a-Si, including the average Si-Si angle distortions and the strain free energy, without predefined threshold owing to its unsupervised nature. This, in turn, facilitates to set a paradigm to categorize the highly disordered structure for amorphous materials. The multiresolution capability of the MML algorithm allows for mining ultra-low abundance structural information at a finer resolution grid. As a result, a new Raman mode of a-Si surface chemistry embedded in a large TERS dataset can be detected. These insights are valuable for unraveling the nanoscale structure, such as defects of semiconductor devices in both fundamental research and industrial applications.

While current study solely focuses on a-Si thin film, the combination of ultra-sensitive surface spectroscopy, TERS and the efficient multiresolution manifold learning algorithm should boost scientific discoveries in a broad scope of disciplines, such as solid-state electrolytes, metal-organic framework (MOF) and low-dimension materials, revealing the unknown unknowns to material and domain scientists.

\section{Methods}

\section{TERS setup and measurements}

A physical vapor deposition (PVD) method was used to fabricate TERS probes from commercial AFM tips (Bruker, OTESPA-R3, resonance frequency $=300 \mathrm{kHz}$, spring constant $=26 \mathrm{~N} / \mathrm{m}$, tip apex diameter $=7$ $\mathrm{nm})$ as reported in a previous study. ${ }^{18}$ Briefly, three sequential depositions of chromium (Cr) $(2 \mathrm{~nm}$ adhesion layer), silver (Ag, plasmonic layer, $\sim 40 \mathrm{~nm}$ ), and aluminum (Al, protection layer, $2 \mathrm{~nm}$ ) were 
performed. Al converts to a dense alumina that provides good mechanical and chemical protection without influencing significantly on tip optical properties. TERS tip fabrication details can be found in Reference ${ }^{58}$.

All TERS measurements were performed on an atomic force microscope (AFM, AIST-NT SMART PROBE) in connection with a Raman spectrometer (HORIBA Co., Xplore) in an argon-filled glove box. For Raman measurements, the $532 \mathrm{~nm}$ laser wavelength was chosen with a local power density of $25 \mu \mathrm{W}$. The grating number was 600 grooves $/ \mathrm{mm}$, and the objective was $100 \times($ N.A. $=0.7$ ). The tapping mode was chosen with oscillation amplitude of $20 \mathrm{~nm}$ and a $\sim 2 \mathrm{~nm}$ minimum distance from the sample surface for AFM. The mapped area was set at $1 \times 1 \mu \mathrm{m}^{2}$ with a step size $20 \mathrm{~nm}$. The accumulation time was $0.5 \mathrm{~s}$ for each spectral acquisition. Each frame of TERS map represents the intensity (after background correction) of the corresponding vibrational mode that arises from a-Si.

\section{FDTD simulation}

Three-dimensional (3D) finite-difference time-domain (FDTD) simulations were used to study the electromagnetic (EM-field) distribution between the tip apex and the a-Si sample. The method was detailed in our previous report, ${ }^{18}$ and elaborated in supporting information (Figure S1).

\section{Manifold Clustering}

Low-dimensional manifold embedding for TERS measurements is calculated via a modified GraphBootstrapping approach. ${ }^{25,26} \mathrm{Graph}$-Bootstrapping is an iterative procedure that consists of two main steps: construction of nearest neighbor graph and manifold layout of nearest neighbor graph. During the initialization (iteration 0) of Graph-Bootstrapping, a nearest neighbor graph is calculated based on the high-dimensional TERS measurements, which we call this graph as root graph. Accordingly, we refer the manifold layout of the root graph as root manifold. During the iteration of Graph-Bootstrapping, nearest neighbor graph is reconstructed based on the low-dimensional manifold coordinates of the previous iteration and subsequently manifold layout is updated based on the newly reconstructed graph.

Graph construction and manifold layout follows the way of LargeVis. ${ }^{59}$ Approximate nearest neighbor graph construction is calculated via random projection tree ${ }^{60}$ and neighbor exploring ${ }^{61}$ techniques, given the input dataset $X=\{X 1, X 2, \ldots . ., X n\} \subseteq R^{d}$. (Recall that, during the initialization of Graph-Bootstrapping procedure, the input dataset $X$ is the original TERS measurements of high dimensionality. During the iterations of Graph-Bootstrapping, the input dataset $X$ is the low-dimensional $(d=2)$ manifold coordinates of the graph of previous iteration). Specifically, the graph is firstly constructed by searching $k$ nearest neighbors via random projection tree method. The graph is then refined via neighbor exploring procedure: 1) Create the max-heap $\mathrm{H}_{\mathrm{i}}$ for each node $\mathrm{i}$ in the graph; 2 ) For each neighbor node $\mathrm{j}$ of node $\mathrm{i}$, calculate Euclidean distances between node i and each neighbor node I of node j, dist $(\mathrm{i}, \mathrm{I})=; 3$ ) Push I with dist $(\mathrm{i}, \mathrm{I})$ into $\mathrm{H}_{\mathrm{i}}$; 4) Pop if $\mathrm{H}_{\mathrm{i}}$ has more than $k$ nodes. For each node $\mathrm{i}$ and each neighbor node $\mathrm{j}$ of $\mathrm{i}$, an 
edge $E(i, j)$ is added to the graph. The weight of symmetric edge $E(i, j)$ is defined in a similar way of $t$-sne method: 62

$$
w_{i j}=\frac{p_{j \mid i}+p_{i \mid j}}{2 n}, p_{j \mid i}=\frac{\exp \left(-\left\|x_{i}-x_{j}\right\|^{2} / 2 \delta_{i}^{2}\right)}{\sum_{(i, k \in E)} \exp \left(-\left\|x_{i}-x_{k}\right\|^{2} / 2 \delta_{i}^{2}\right)}
$$

To calculate a low-dimensional manifold layout of the graph where each node $\mathrm{i}$ of graph is represented by a point $y_{i}$ in 2D space, a likelihood function is constructed to preserve pair-wise similarities of the nodes in the $2 \mathrm{D}$ space, 59

$$
L\left(y_{1}, y_{2}, \ldots \ldots, y_{n}\right)=\prod_{(i, j) \in E}\left[f\left(\left\|y_{i}-y_{j}\right\|^{2}\right)\right]^{w_{i j}} \prod_{(i, j) \in \bar{E}}\left[1-f\left(\left\|y_{i}-y_{j}\right\|^{2}\right)\right]^{\epsilon}
$$

Equation (4)

where $\mathrm{f}$ is a probability function set as $f(x)=\frac{1}{1+x^{2}}$ and $\epsilon$ is a unified weight. Intuitively, first part of above equation will keep similar nodes close in 2D space meanwhile second part will tell apart dissimilar nodes in 2D space. The likelihood function can be efficiently maximized with respect to $\left(y_{1}, y_{2}, \ldots \ldots, y_{n}\right)$ via negative sampling ${ }^{63}$ and alias table sampling ${ }^{64}$ of unfolded weighted edges, ${ }^{65}$ followed by asynchronous stochastic gradient descent. ${ }^{66}$ During implementations, we set all hyperparameters default as in LargeVis ${ }^{59}$ without any signal preprocessing.

Clustering on manifold is performed by hierarchical density estimates method (HDBSCAN), ${ }^{67}$ that relies on the mutual reachability distance:

$D_{\text {mreach, },}(a, b)=\max \left\{\operatorname{core}_{k}(a), \operatorname{core}_{k}(b), d(a, b)\right\}$,

where $d(a, b)$ is the original metric distance (Euclidean distance in this paper) between points $a$ and $b$, core $_{k}(x)$ is the core-distance of a point $x$ to cover its $k$ nearest neighbors. A minimum spanning tree is firstly built and then condensed upon the hyper-parameter of minimum cluster size, $m c$. The stability of each cluster $C_{i}$ is defined as:

$$
S\left(C_{i}\right)=\sum_{a \in C_{i}} \lambda_{\max , C_{i}, a}-\lambda_{\min , C_{i}, a}
$$

where $\lambda$ is the reciprocal of core-distance, $\lambda_{\max , C_{i} a}$ is the $\lambda$ value at which point $a$ falls out of cluster $C_{i}$ and $\lambda_{\min , \mathrm{C}_{i} \mathrm{a}}$ is the minimum $\lambda$ value at which point $a$ is present in $\mathrm{C}_{\mathrm{i}}$. Optimal flat clusters are extracted via walking up the tree to maximize the total stability score over chosen clusters: considering all leaf nodes as initial clusters, if the cluster' stability is greater than the sum of its child, the cluster is selected to be in current set of optimal flat clustering and all its child are removed from the set. Otherwise, the cluster's stability is set to be the sum of its child stabilities. The main tuning parameter is the minimum cluster 
size, $m c$. We leave all the other tuning parameters of HDBSCAN as default. For the parent manifold clusters in Figure 3 , we follow a similar procedure in ref. ${ }^{21}$ to choose $m c$. We first consider all integer $m c$ values in a wide range of $[10,150]$. We then fit the trend of total number of estimated clusters against every $m c$ value by the exponential decay function. We choose the $m c$ in the tail region where the total number of clusters tends to be stable. For child manifold clusters at the finer resolution grid as shown in Figure 5 , we set the $m c$ a small value around $0.5 \%$ of the total number of TERS measurements.

\section{Neutron Scattering}

All neutron scattering measurements were performed at the Spallation Neutron Source at Oak Ridge National Laboratory (ORNL). Prior to each measurement, the a-Si film was peeled off from the copper substrate in the Ar-filled glovebox $\left(\mathrm{O}_{2}<0.1 \mathrm{ppm}, \mathrm{H}_{2} \mathrm{O}<0.1 \mathrm{ppm}\right)$ and sealed in a vanadium can $(\varnothing=6$ $\mathrm{mm}$ ). The total amount of a-Si was $1.368 \mathrm{~g}$ and the height was $3.9 \mathrm{~cm}$. An empty vanadium can of the same type was sealed in Ar-glovebox and used as a blank reference.

1. Neutron total scattering structure function. The structure function data was collected at NanoscaleOrdered Materials Diffractometer (NOMAD) beamline per a procedure published by Neuefeind et al. ${ }^{68}$ The data collection time was $150 \mathrm{~min}$. The structure factor, $\mathrm{S}(\mathrm{Q})$ was obtained from a $\mathrm{Q}$ range between 0.5 and $31 \AA^{-1}$.

2. Inelastic neutron scattering (INS). INS spectra were obtained at the VISION beamline on the same aSi measured by neutron PDF to assure consistency. The sample was measured in vanadium sample holder at $5 \mathrm{~K}$ for about 10 hours. The empty sample holder was also measured, and the background spectrum was removed to obtain the spectrum from the sample.

\section{Molecular Dynamics (MD) simulations}

The models of a-Si were established by conducting molecular dynamics simulations in LAMMPS. ${ }^{69}$ Following a melting-and-quench procedure, ${ }^{70}$ the initial network a-Si was firstly annealed to $2400 \mathrm{~K}$ in the $N V T$ ensemble, and then quenched to room temperature $(300 \mathrm{~K})$ at a quench rate of $10^{12} \mathrm{Ks}^{-1}$. The a-Si models were finally fully relaxed at $300 \mathrm{~K}$ in the NPT ensemble. The SW-VBM interatomic potential ${ }^{71}$ was used to describe Si-Si interaction. The average coordination number of constructed a-Si models is $\sim 3.99$ and quite close to the experimental value $(\sim 3.8-3.9),{ }^{72,73,74}$ validating the high quality of these a-Si models.

\section{Density Functional Theory (DFT) calculations}

Density Functional Theory (DFT) modeling was performed using the Vienna ab initio Simulation Package (VASP)..$^{75}$ The calculation used Projector Augmented Wave (PAW) method ${ }^{76,77}$ to describe the effects of core electrons, and Perdew-Burke-Ernzerhof (PBE) ${ }^{78}$ implementation of the Generalized Gradient Approximation (GGA) for the exchange-correlation functional. Energy cutoff was $600 \mathrm{eV}$ for the planewave basis of the valence electrons. The starting structure of a-Si slab with both surfaces terminated by 
$-\mathrm{H}$ and $-\mathrm{OH}$ was generated by MD simulations as discussed above. ${ }^{69}$ The thickness of the slab is about $1.5 \mathrm{~nm}$, and the total thickness of the simulation box is $3.5 \mathrm{~nm}$ (i.e., $2 \mathrm{~nm}$ vacuum). The surface area is about $1.05 \mathrm{~nm} \times 1.05 \mathrm{~nm}$. The simulation box contains $64 \mathrm{Si}$ atoms, and is under 3D periodic boundary condition. The electronic structure was calculated on a $3 \times 3 \times 1$ Monkhorst-Pack mesh. The total energy tolerance for electronic energy minimization was $10^{-5} \mathrm{eV}$, and the maximum interatomic force after relaxation was below $0.01 \mathrm{eV} / \AA \AA$. The vibrational eigen-frequencies and modes were then calculated by the finite displacement method. The OClimax software ${ }^{79}$ was used to convert the DFT-calculated phonon results to the simulated INS spectra.

\section{Declarations}

\section{Acknowledgments}

This work is supported by the U.S. Department of Energy's Vehicle Technologies Office under the Silicon Electrolyte Interface Stabilization (SEISta) Consortium directed by Brian Cunningham and managed by Anthony Burrell. Part of this work was supported by The work was supported by the Center for Nanophase Materials Sciences, a U.S. Department of Energy, Office of Science User Facility at Oak Ridge National Laboratory (S.V.K), Division of Materials Science and Engineering, Biomolecular Materials Program, and Energy Frontier Research Center CSSAS located at University of Washington (X.L.). We thank Dr. Dmitry N. Voylov for extremely useful discussion on the TERS experimental setup. We are grateful for fruitful discussions on RF sputtering with Drs. Nancy J. Dudney, Andrew Kercher, and Robert L. Sacci. We thank Drs. Andrew Westover, Jue Liu and Katharine L. Page discussion for NOMAD setup. We thank Michelle S. Everett for strong technical support for NOMAD.

\section{References}

1. Chen R, et al. Nanophotonic integrated circuits from nanoresonators grown on silicon. Nature communications 5, 1-10 (2014).

2. Vivien L, Pavesi L. Handbook of silicon photonics. Taylor \& Francis (2016).

3. Ndiaye A, Charki A, Kobi A, Kébé CM, Ndiaye PA, Sambou V. Degradations of silicon photovoltaic modules: A literature review. Solar Energy 96, 140-151 (2013).

4. Kim J, et al. $10.5 \%$ efficient polymer and amorphous silicon hybrid tandem photovoltaic cell. Nature communications 6, 1-6 (2015).

5. Hou T, et al. The influence of FEC on the solvation structure and reduction reaction of LiPF6/EC electrolytes and its implication for solid electrolyte interphase formation. Nano Energy 64, 103881 (2019).

6. Wu $\mathrm{H}$, et al. Stable cycling of double-walled silicon nanotube battery anodes through solidelectrolyte interphase control. Nature nanotechnology 7, 310 (2012). 
7. Ryu J, et al. Infinitesimal sulfur fusion yields quasi-metallic bulk silicon for stable and fast energy storage. Nature communications 10, 1-9 (2019).

8. Hasan M, Huq MF, Mahmood ZH. A review on electronic and optical properties of silicon nanowire and its different growth techniques. SpringerPlus 2, 151 (2013).

9. Kane BE. A silicon-based nuclear spin quantum computer. nature 393, 133 (1998).

10. Russell JP. Raman scattering in silicon. Applied Physics Letters 6, 223-224 (1965).

11. Parker Jr J, Feldman D, Ashkin M. Raman scattering by silicon and germanium. Physical Review 155, 712 (1967).

12. Richter $\mathrm{H}$, Wang $\mathrm{Z}$, Ley L. The one phonon Raman spectrum in microcrystalline silicon. Solid State Communications 39, 625-629 (1981).

13. Yue G, Lorentzen J, Lin J, Han D, Wang Q. Photoluminescence and Raman studies in thin-film materials: Transition from amorphous to microcrystalline silicon. Applied Physics Letters 75, 492494 (1999).

14. De Wolf I. Micro-Raman spectroscopy to study local mechanical stress in silicon integrated circuits. Semiconductor science and technology 11, 139 (1996).

15. Zeng Z, Liu N, Zeng Q, Lee SW, Mao WL, Cui Y. In situ measurement of lithiation-induced stress in silicon nanoparticles using micro-Raman spectroscopy. Nano Energy 22, 105-110 (2016).

16. Perichon S, Lysenko V, Remaki B, Barbier D, Champagnon B. Measurement of porous silicon thermal conductivity by micro-Raman scattering. Journal of Applied Physics 86, 4700-4702 (1999).

17. Wang X, Huang S-C, Hu S, Yan S, Ren B. Fundamental understanding and applications of plasmonenhanced Raman spectroscopy. Nature Reviews Physics, 1-19 (2020).

18. Nanda J, et al. Unraveling the Nanoscale Chemical Heterogeneity of Solid Electrolyte Interphase using Tip-Enhanced Raman Spectroscopy (in press). Joule, (2019).

19. Yano T-a, et al. Tip-enhanced nano-Raman analytical imaging of locally induced strain distribution in carbon nanotubes. Nature communications 4, 1-7 (2013).

20. Sonntag MD, Pozzi EA, Jiang N, Hersam MC, Van Duyne RP. Recent advances in tip-enhanced Raman spectroscopy. J Phys Chem Lett 5, 3125-3130 (2014).

21. Sun W, Shen Z. A practical nanoscopic Raman imaging technique realized by near-field enhancement. Materials Physics and Mechanics 4, 17-21 (2001).

22. Lee $\mathrm{N}$, et al. High contrast scanning nano-Raman spectroscopy of silicon. Journal of Raman Spectroscopy: An International Journal for Original Work in all Aspects of Raman Spectroscopy, Including Higher Order Processes, and also Brillouin and Rayleigh Scattering 38, 789-796 (2007).

23. Brockhouse B. Lattice vibrations in silicon and germanium. Physical Review Letters 2, 256 (1959).

24. Zhong $\mathrm{M}$, et al. Accelerated discovery of $\mathrm{CO} 2$ electrocatalysts using active machine learning. Nature 581, 178-183 (2020).

25. Li X, Collins L, Miyazawa K, Fukuma T, Jesse S, Kalinin SV. High-veracity functional imaging in scanning probe microscopy via Graph-Bootstrapping. Nature communications 9, 2428 (2018). 
26. Li X, et al. Manifold learning of four-dimensional scanning transmission electron microscopy. $n p j$ Computational Materials 5, 5 (2019).

27. Morell G, Katiyar R, Weisz S, Jia H, Shinar J, Balberg I. Raman study of the network disorder in sputtered and glow discharge a-Si: H films. Journal of applied physics 78, 5120-5125 (1995).

28. Tsu R, Gonzalez-Hernandez J, Pollak FH. Determination of the energy barrier for structural relaxation in amorphous Si and Ge by Raman scattering. Solid state communications 54, 447-450 (1985).

29. Zallen R. The physics of amorphous solids. John Wiley \& Sons (2008).

30. Morimoto A, Ooroza S, Kumeda M, Shimizu T. Raman studies on local structural disorder in siliconbased amorphous semiconductor films. Solid state communications 47, 773-777 (1983).

31. Voutsas A, Hatalis M, Boyce J, Chiang A. Raman spectroscopy of amorphous and microcrystalline silicon films deposited by low-pressure chemical vapor deposition. Journal of Applied Physics 78, 6999-7006 (1995).

32. Tsu R, Gonzalez-Hernandez J, Doehler J, Ovshinsky S. Order parameters in a-Si systems. Solid state communications 46, 79-82 (1983).

33. Sinke W, Roorda S, Saris F. Variable strain energy in amorphous silicon. Journal of Materials Research 3, 1201-1207 (1988).

34. Vink R, Barkema G, Van Der Weg W. Raman spectra and structure of amorphous Si. Physical Review $B$ 63, 115210 (2001).

35. Renucci J, Tyte R, Cardona M. Resonant Raman scattering in silicon. Physical Review B 11, 3885 (1975).

36. Treacy M, Borisenko K. The local structure of amorphous silicon. Science 335, 950-953 (2012).

37. Brodsky M, Cardona M. Local order as determined by electronic and vibrational spectroscopy: amorphous semiconductors. Journal of Non-Crystalline Solids 31, 81-108 (1978).

38. Alben R, Weaire D, Smith Jr J, Brodsky M. Vibrational properties of amorphous Si and Ge. Physical Review B 11, 2271 (1975).

39. Keating P. Effect of invariance requirements on the elastic strain energy of crystals with application to the diamond structure. Physical Review 145, 637 (1966).

40. Tsu R. Structural characterization of amorphous silicon and germanium. In: Disordered Semiconductors). Springer (1987).

41. Saito T, Karasawa T, Ohdomari I. Distortion energy distributions in the random network model of amorphous silicon. Journal of Non-Crystalline Solids 50, 271-276 (1982).

42. Steinhardt P, Alben R, Weaire D. Relaxed continuous random network models:(I). Structural characteristics. Journal of Non-Crystalline Solids 15, 199-214 (1974).

43. Vignoli S, Mélinon P, Masenelli B, i Cabarrocas PR, Flank A, Longeaud C. Over-coordination and order in hydrogenated nanostructured silicon thin films: their influence on strain and electronic properties. Journal of Physics: Condensed Matter 17, 1279 (2005). 
44. Deringer VL, et al. Realistic atomistic structure of amorphous silicon from machine-learning-driven molecular dynamics. The journal of physical chemistry letters 9, 2879-2885 (2018).

45. Barkema G, Mousseau N. Event-based relaxation of continuous disordered systems. Physical review letters 77, 4358 (1996).

46. Mousseau N, Barkema G. Traveling through potential energy landscapes of disordered materials: The activation-relaxation technique. Physical Review E 57, 2419 (1998).

47. Marinov M, Zotov N. Model investigation of the Raman spectra of amorphous silicon. Physical review $B$ 55, 2938 (1997).

48. Beeman D, Tsu R, Thorpe M. Structural information from the Raman spectrum of amorphous silicon. Physical Review B 32, 874 (1985).

49. Squires GL. Introduction to the theory of thermal neutron scattering. Cambridge university press (2012).

50. Sears VF. Neutron scattering lengths and cross sections. Neutron news 3, 26-37 (1992).

51. Volodin V, Koshelev D. Quantitative analysis of hydrogen in amorphous silicon using Raman scattering spectroscopy. Journal of Raman spectroscopy 44, 1760-1764 (2013).

52. Brodsky M, Cardona M, Cuomo J. Infrared and Raman spectra of the silicon-hydrogen bonds in amorphous silicon prepared by glow discharge and sputtering. Physical Review B 16, 3556 (1977).

53. Harrelson TF, et al. Identifying atomic scale structure in undoped/doped semicrystalline p3ht using inelastic neutron scattering. Macromolecules 50, 2424-2435 (2017).

54. Tsu D, Lucovsky G, Davidson B. Effects of the nearest neighbors and the alloy matrix on $\mathrm{SiH}$ stretching vibrations in the amorphous SiO $\mathrm{r}: \mathrm{H}(0<\mathrm{r}<2)$ alloy system. Physical Review B 40, 1795 (1989).

55. Lucovsky G. Chemical effects on the frequencies of Si-H vibrations in amorphous solids. Solid State Communications 29, 571-576 (1979).

56. Sanderson RT. Chemical periodicity. Reinhold Pub. Corp. (1960).

57. Borghesi A, Guizzetti G, Sassella A, Bisi O, Pavesi L. Induction-model analysis of Siî冈, H stretching mode in porous silicon. Solid state communications 89, 615-618 (1994).

58. Barrios CA, Malkovskiy AV, Kisliuk AM, Sokolov AP, Foster MD. Highly stable, protected plasmonic nanostructures for tip enhanced Raman spectroscopy. The Journal of Physical Chemistry C 113, 8158-8161 (2009).

59. Tang J, Liu J, Zhang M, Mei Q. Visualizing large-scale and high-dimensional data. In: Proceedings of the 25th international conference on world wide web). International World Wide Web Conferences Steering Committee (2016).

60. Dasgupta S, Freund Y. Random projection trees and low dimensional manifolds. In: STOC). Citeseer (2008).

61. Dong W, Moses C, Li K. Efficient k-nearest neighbor graph construction for generic similarity measures. In: Proceedings of the 20th international conference on World wide web). ACM (2011). 
62. Maaten Lvd, Hinton G. Visualizing data using t-SNE. Journal of machine learning research 9, 25792605 (2008).

63. Mikolov T, Sutskever I, Chen K, Corrado GS, Dean J. Distributed representations of words and phrases and their compositionality. In: Advances in neural information processing systems) (2013).

64. Li AQ, Ahmed A, Ravi S, Smola AJ. Reducing the sampling complexity of topic models. In: Proceedings of the 20th ACM SIGKDD international conference on Knowledge discovery and data mining). ACM (2014).

65. Tang J, Qu M, Wang M, Zhang M, Yan J, Mei Q. Line: Large-scale information network embedding. In: Proceedings of the 24th international conference on world wide web). International World Wide Web Conferences Steering Committee (2015).

66. Recht B, Re C, Wright S, Niu F. Hogwild: A lock-free approach to parallelizing stochastic gradient descent. In: Advances in neural information processing systems) (2011).

67. Campello RJ, Moulavi D, Sander J. Density-based clustering based on hierarchical density estimates. In: Pacific-Asia conference on knowledge discovery and data mining). Springer (2013).

68. Neuefeind J, Feygenson M, Carruth J, Hoffmann R, Chipley KK. The nanoscale ordered materials diffractometer NOMAD at the spallation neutron source SNS. Nuclear Instruments and Methods in Physics Research Section B: Beam Interactions with Materials and Atoms 287, 68-75 (2012).

69. Plimpton S. Fast parallel algorithms for short-range molecular dynamics. Journal of computational physics 117, 1-19 (1995).

70. Sitinamaluwa H, Nerkar J, Wang M, Zhang S, Yan C. Deformation and failure mechanisms of electrochemically lithiated silicon thin films. RSC Advances 7, 13487-13497 (2017).

71. Vink R, Barkema G, Van der Weg W, Mousseau N. Fitting the Stillinger-Weber potential to amorphous silicon. Journal of non-crystalline solids 282, 248-255 (2001).

72. Kugler $S$, et al. Neutron-diffraction study of the structure of evaporated pure amorphous silicon. Physical Review B 40, 8030 (1989).

73. Fortner J, Lannin J. Radial distribution functions of amorphous silicon. Physical Review B 39, 5527 (1989).

74. Roorda S, et al. Structural relaxation and defect annihilation in pure amorphous silicon. Physical review $B$ 44, 3702 (1991).

75. Kresse G, Furthmüller J. Efficient iterative schemes for ab initio total-energy calculations using a plane-wave basis set. Physical review B 54, 11169 (1996).

76. Blöchl PE. Projector augmented-wave method. Physical review B 50, 17953 (1994).

77. Kresse G, Joubert D. From ultrasoft pseudopotentials to the projector augmented-wave method. Physical Review B 59, 1758 (1999).

78. Perdew JP, Burke K, Ernzerhof M. Generalized gradient approximation made simple. Physical review letters 77, 3865 (1996). 
79. Cheng Y, Daemen L, Kolesnikov A, Ramirez-Cuesta A. Simulation of inelastic neutron scattering spectra using OCLIMAX. Journal of chemical theory and computation 15, 1974-1982 (2019).

\section{Figures}

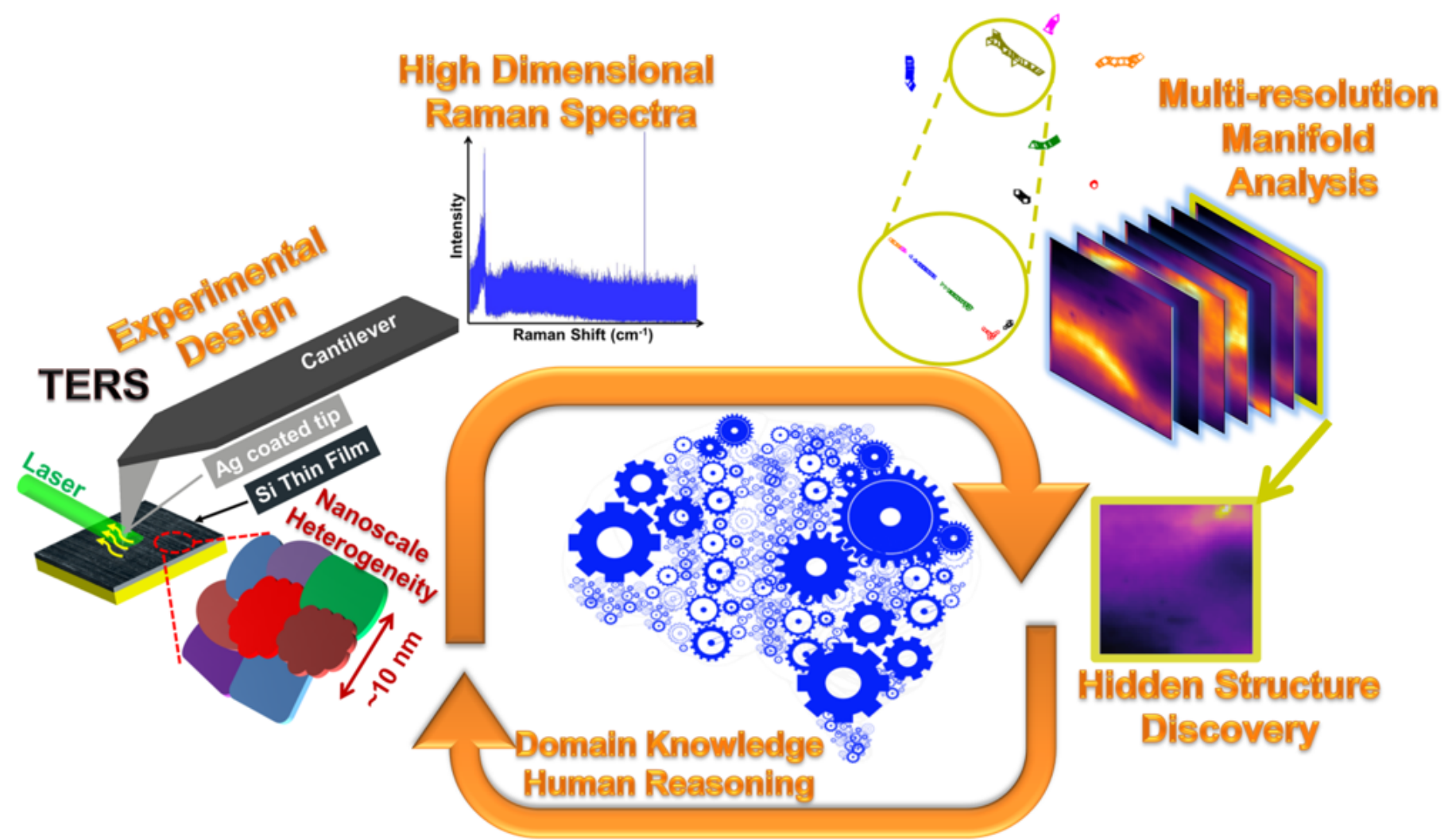

\section{Figure 1}

The integrated workflow comprising hyperspectral TERS imaging and the multi-resolution manifold learning (MML) algorithm to depict a-Si structural heterogeneity at the nanoscale. The low dimensional physical parameters characterizing material structures such as local defects and atomic vibrations are translated into the high dimensional Raman spectra via hyperspectral TERS imaging transfer functions. The intrinsic low dimensionality of the physics suggests the structure of TERS measurements on the sample as a whole can be projected to a low dimensional latent manifold space via MML. Exploration data analysis such as clustering can be efficiently conducted on the low-dimensional manifold space to reveal salient features for evaluating material structure heterogeneity by human reasoning and updating domain knowledge in a loop. 

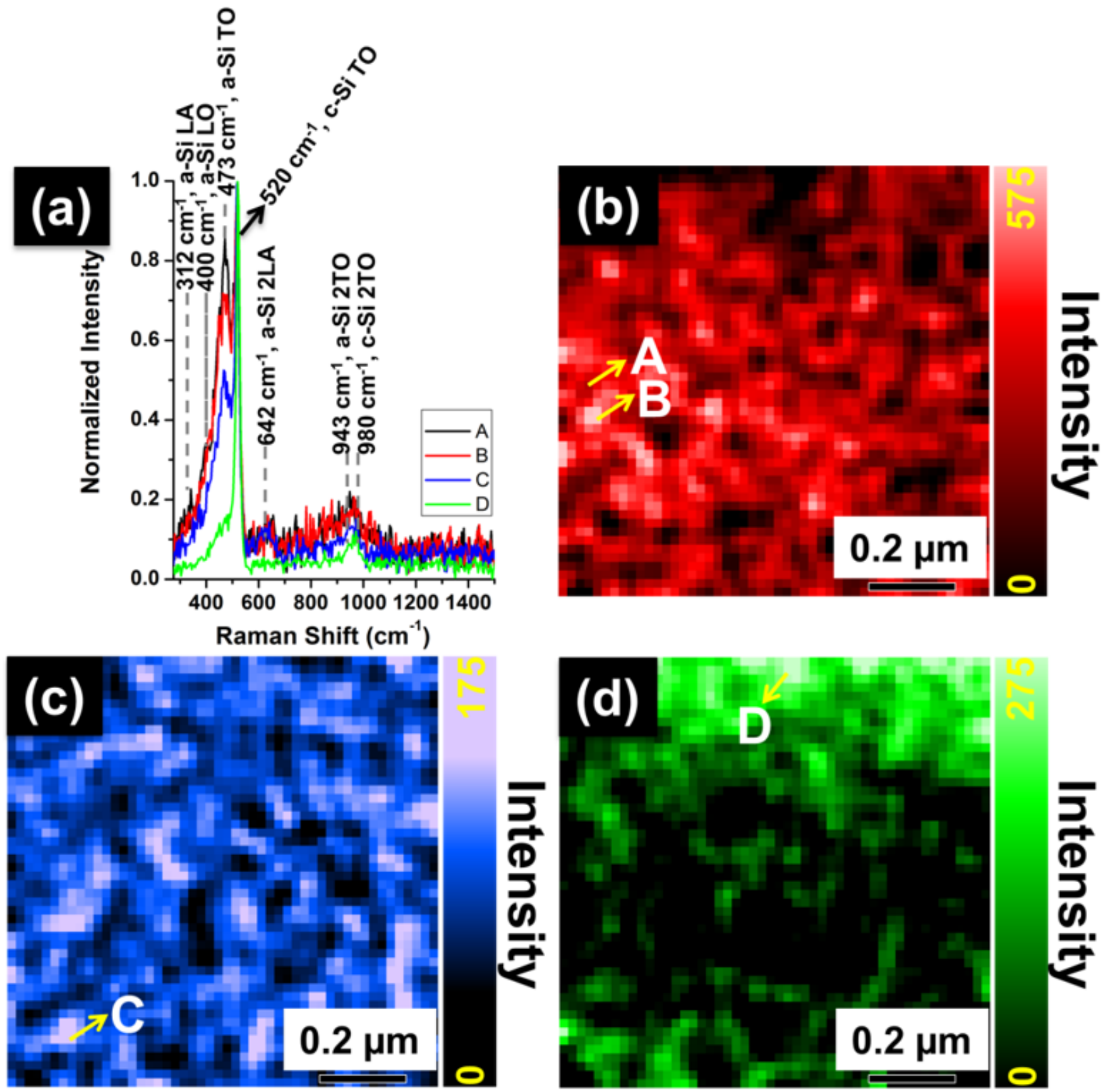

Figure 2

(a) TERS spectra collected from various locations on the a-Si surface labeled by "A" to " $D$ " in (c-d). TERS mapping of an individual peak intensity centered at (b) $473 \mathrm{~cm}-1$ (a-Si TO mode), (c) $642 \mathrm{~cm}-1$ (2LA mode), and (d) $943 \mathrm{~cm}-1$ (2TO mode). A hybrid TERS mapping combining the peak intensity distribution in (b-d) is shown in Figure $S 2$. 

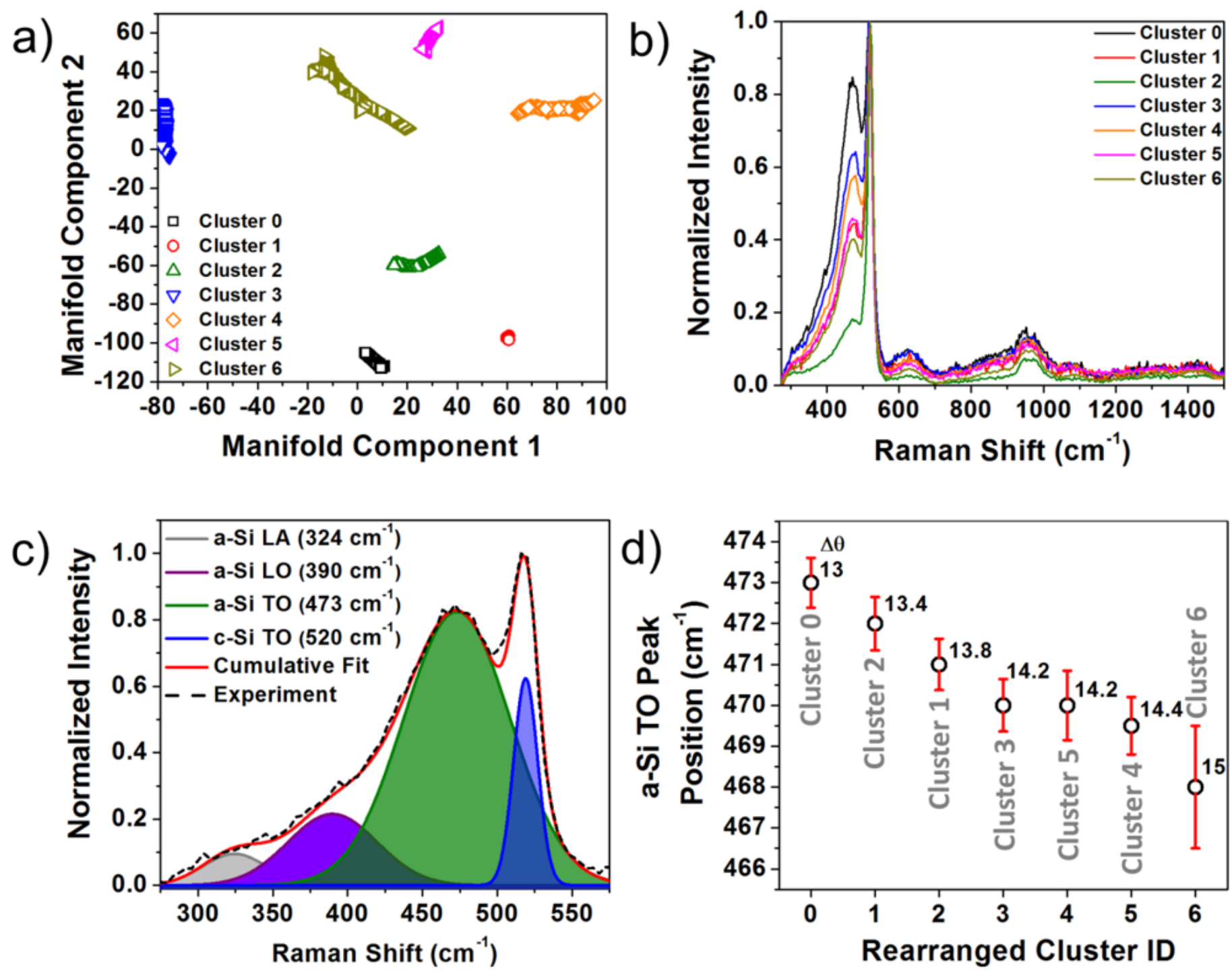

Figure 3

(a) The parent manifold layout via the Graph-Bootstrapping method (see details in Method section) colored by the cluster labels. (b) The mean TERS spectrum for each cluster (spectrum normalized against the c-Si TO band at $520 \mathrm{~cm}-1$ ). (c) Peak deconvolution of the silicon TO modes, LO mode and LA mode.

(d) The TO-band center arranged in declining order. The error bar stands for the Gaussian fitting deviation of the peak center. $\Delta \theta$ is defined as the deviation of the Si-Si bond angle in the a-Si random network from that of the single crystal $\mathrm{Si}(109.50)$. 

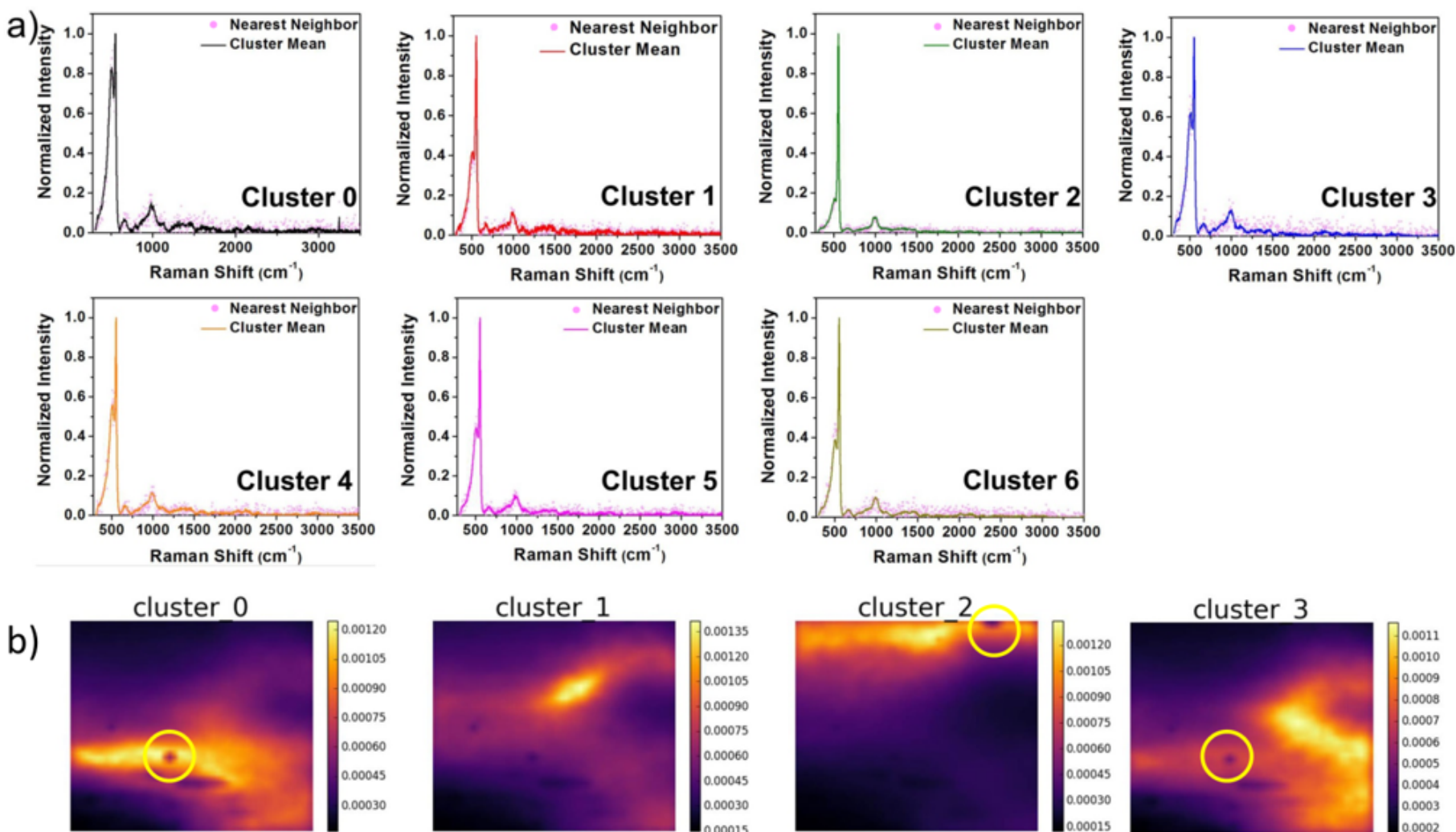

cluster 4
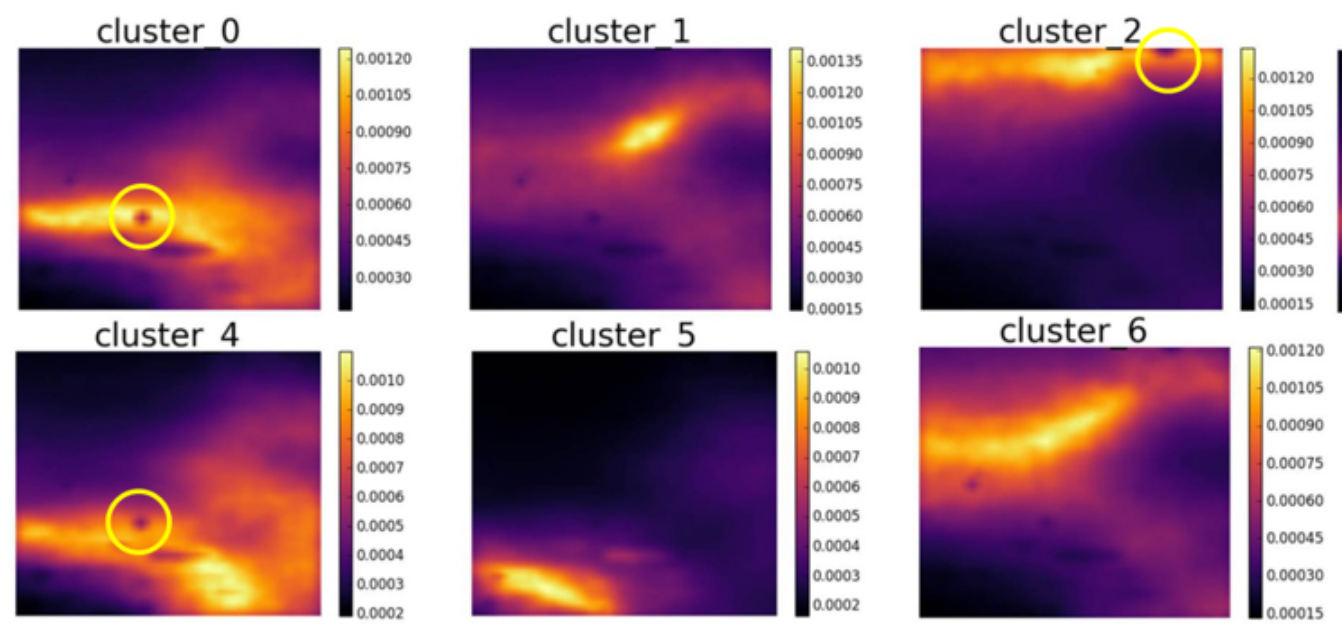

cluster 3
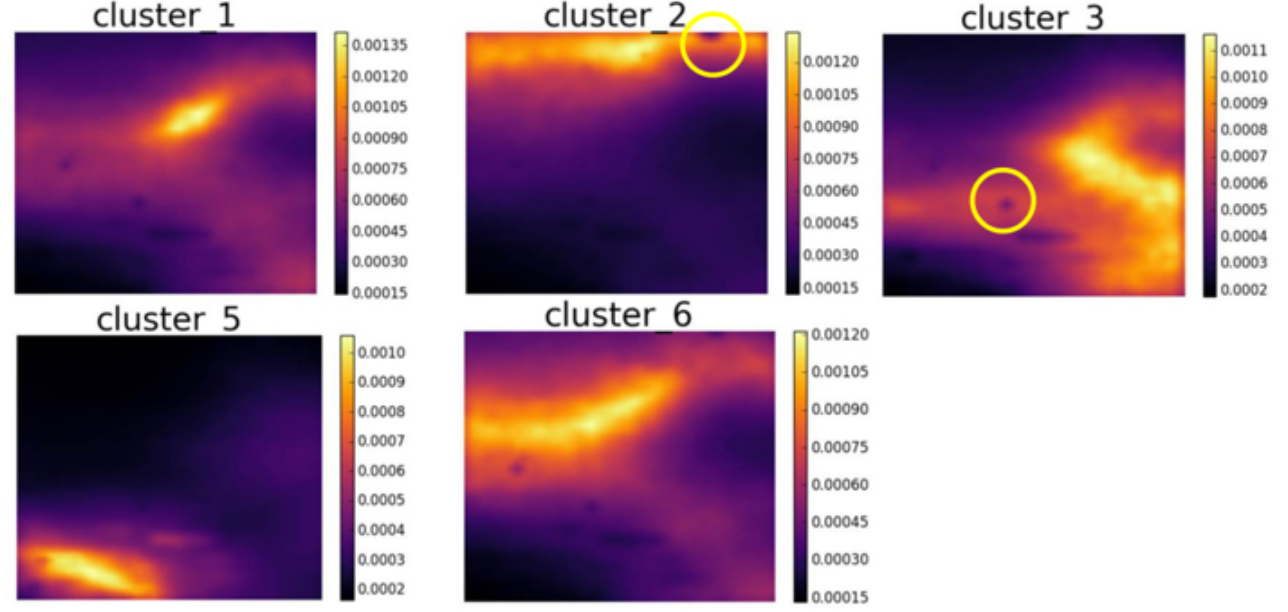

\section{Figure 4}

(a) Average TERS spectrum of each cluster and its nearest neighbor in experimental data. (b) Similarity loading of each cluster. The color bar represents the reciprocal of Euclidean distance between the mean TERS spectrum and the as-taken TERS spectrum of each the pixel within a given cluster. 


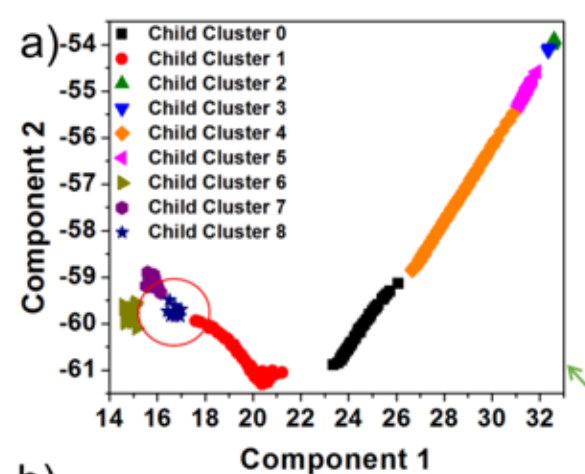

b) 2

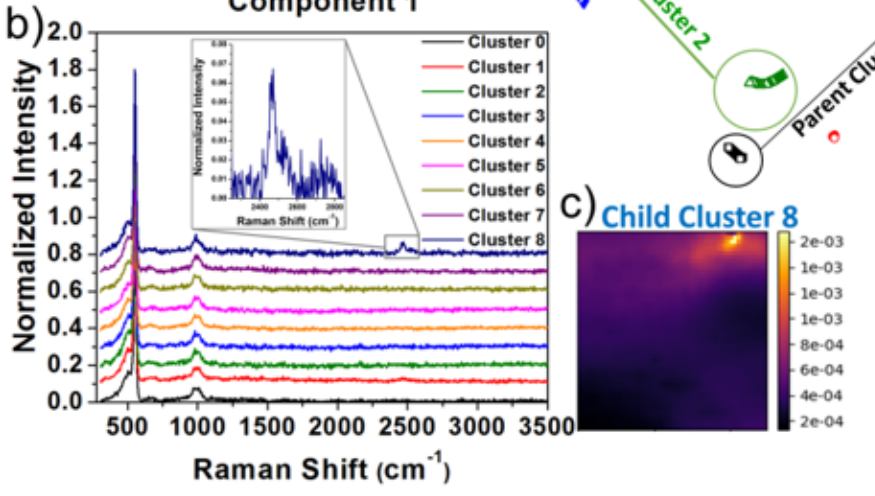

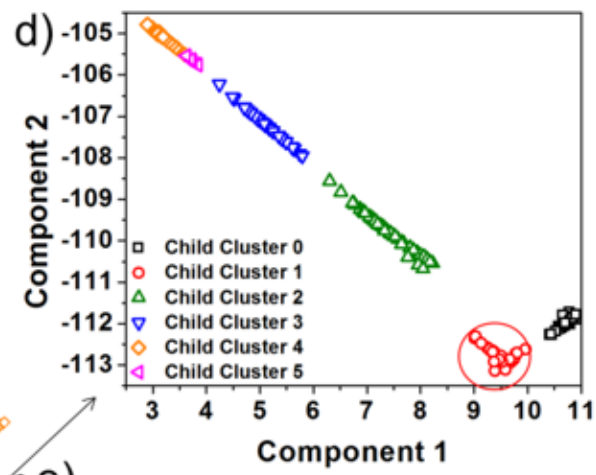

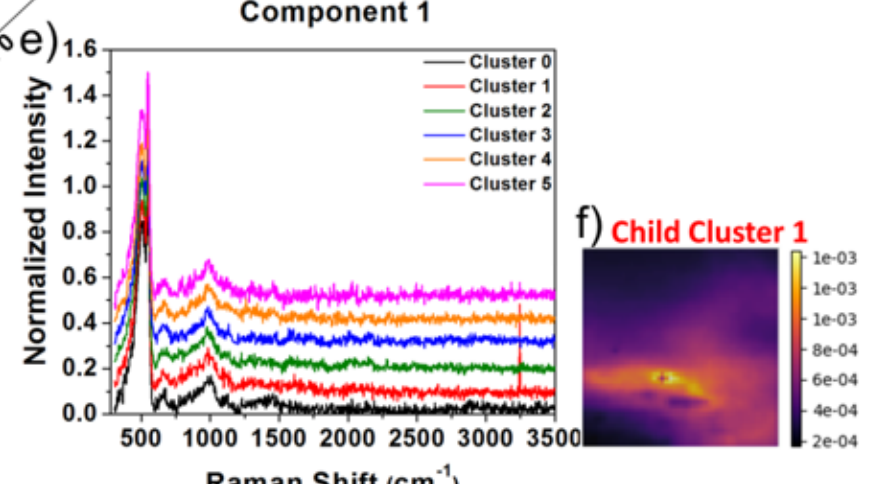

\section{Figure 5}

(a) An overview of child manifold clusters at the finer resolution grid, stemmed from Parent Cluster 2 and (b) the corresponding mean TERS spectra of the child clusters. The peak centered at $2435 \mathrm{~cm}-1$ of Child Cluster 8 represents unique structural defects different from other child clusters. (c) Similarity loading of Child Cluster 8 that shows a bright blob around the black singular patches in similarity loading of Parent Cluster 2 in Figure 4. (d) Overview of child clusters stemmed from Parent Cluster 0 and (e) the corresponding mean Raman spectra of the child clusters. (f) The similarity loading of Child Cluster 1 that shows a bright blob around the black singular patches in Parent Cluster 0 similarity loading in Figure 4.
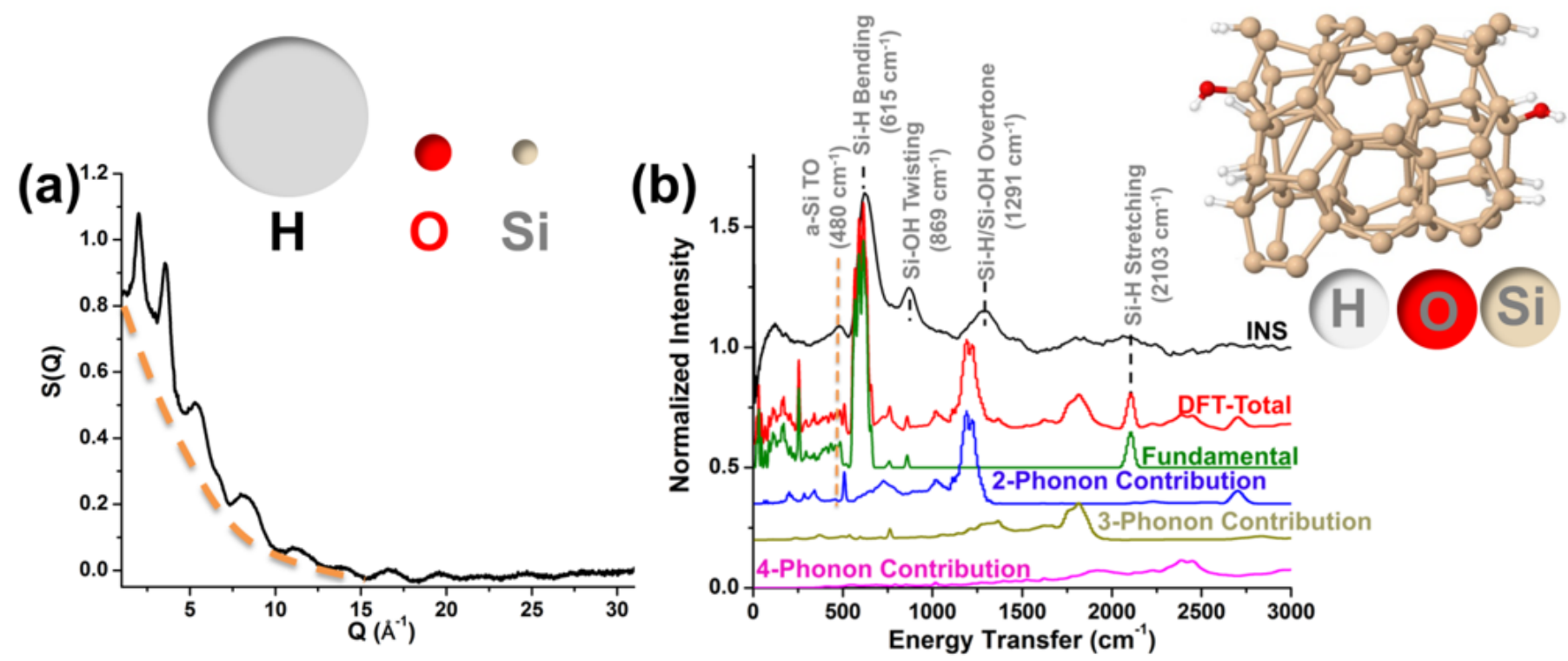


\section{Figure 6}

(a) Neutron scattering plot of the structure factor, $\mathrm{S}$ versus the scattering vector, $\mathrm{Q}$ collected from a-Si under the same sputtering condition to deposit the a-Si thin film. The dash curve guides the eye as an indication of the background from incoherent scattering. Inset schematically illustrates the relative size of the incoherent scattering cross section (XS) of proton (80.260), the coherent XS of oxygen (4.232) and the coherent XS of silicon (2.163). (b) Comparison between the experimental inelastic neutron scattering spectrum of the a-Si sample and that calculated using DFT based on the atomic conformations shown in the inset image. The calculated total spectrum combines the fundamental vibrational modes and higher order excitations up to 10 orders.

\section{Supplementary Files}

This is a list of supplementary files associated with this preprint. Click to download.

- 20200622SIcleanedGYJagjitNanda.docx 